\title{
1-Butyl-3-Methylimidazolium Chloride Ionic Liquid as Inhibitor for Corrosion Protection of Mild Steel in Acidic Media
}

\author{
A.A. Ahmad Tarmizi*, L. Peter ${ }^{2}$, D. Ahtoi ${ }^{2}$, R. Yusof ${ }^{2}$, S.N.A. Syed Ismail ${ }^{2}$ and D.A. Tholibon ${ }^{3}$ \\ ${ }^{l}$ Department of Chemistry, Faculty of Applied Sciences, Universiti Teknologi Mara Shah Alam, 40450, Shah Alam, \\ Selangor, Malaysia \\ ${ }^{2}$ Chemistry Department, Faculty of Applied Sciences, Universiti Teknologi MARA Perlis, 02600 Arau, Perlis, Malaysia \\ ${ }^{3}$ Faculty of Civil Engineering, Universiti Teknologi MARA Pahang, Kampus Jengka, 26400 BandarJengka, Pahang, Malaysia \\ *Corresponding author E-mail: amirahamalina@ uitm.edu.my
}

\begin{abstract}
Corrosion inhibitor is a substance added to the corrosive environment in small quantities to reduce the corrosion a metal. The addition of inhibitors will reduce the corrosion rate of the metal by retarding the corrosion process on the metal surface. Thus, this study focused on the study of the effectiveness of 1- butyl-3-methylimidazolium chloride ([EMIM]Cl) ionic liquid as an inhibitor for corrosion protection of mild steel. Two different concentrations of [EMIM]Cl $(0.05 \mathrm{M}$ and $0.5 \mathrm{M})$ were tested into different concentrations of sulphuric acid $(0.05 \mathrm{M}, 0.10 \mathrm{M}, 0.15 \mathrm{M}, 0.20 \mathrm{M}$ and $0.25 \mathrm{M})$. Weight loss measurement was used to determine the effectiveness of the [EMIM]Cl as inhibitor for corrosion protection. Results showed that acid concentrations play an important role for the corrosion protection process in the presence of the inhibitor. The weight loss increases as the concentration of the acid increased. The study also revealed that the concentrations of [EMIM]Cl ionic liquid effect the performance of the inhibitor. From this study, $0.5 \mathrm{M}$ of [EMIM]Cl ionic liquid shows better corrosion performance compared to $0.05 \mathrm{M}$ of [EMIM]Cl. At $0.25 \mathrm{M}$ of sulphuric acid, the weight loss of untreated metal increased drastically from $0.0075 \mathrm{~g}$ at 0.5 hour to $0.0974 \mathrm{~g}$ at 24 hours. After treated with $0.5 \mathrm{M}$ of [EMIM]Cl, the weight loss measurement slightly increased from $0.0027 \mathrm{~g}$ at 0.5 hour to $0.0179 \mathrm{~g}$ at 24 hours. This weight loss value is lower compared to mild steel treated with $0.05 \mathrm{M}$ [EMIM]Cl which is $0.0469 \mathrm{~g}$ at 24 hours. The performance of the inhibitor in two different type of acid was also investigated. The morphology of the untreated mild steel and mild steel treated with [EMIM]Cl was investigated by scanning electronic microscopy (SEM)
\end{abstract}

Keywords: ionic liquid, inhibitor, acidic media, corrosion, weight loss.

\section{Introduction}

Corrosion is a destructive attack to a material by reaction with its environment. The corrosion occurs due to the compounds directly interact with the metal in different medium or conditions. The spontaneous process which is electrically contact between anodic, cathodic and electrolyte also contribute to corrosion and known as electrochemical corrosion. The corrosion issues lead the attention of scientist to study on how to overcome the corrosion phenomenon.

It was well known that acidic condition promotes corrosion process on mild steel especially in the environment with high concentration of acid. Velrani et al. [1], reported that organic compound act as a good inhibitor in acidic media. Acid's inhibitor is mostly known as organic compounds which contained nitrogen $(\mathrm{N})$, oxygen $(\mathrm{O})$ and sulphur (S) atoms. Previous study $[2,3,4]$ reported that compounds containing $\mathrm{N}, \mathrm{O}$ and $\mathrm{S}$ were proven to be effective inhibitors for the corrosion protection of mild steel in the acidic media. Recently, researchers suggested ionic liquid as one of the effective metallic inhibitors. Researcher prefer to use ionic liquid as metallic due to their physical properties. Ionic liquids are nontoxic nature, low volatility and there are chemically stable. Researchers also claimed ionic liquid as "green corrosion inhibitor".
Ionic liquids (ILs) refer to organic salts with melting points at or below room temperature [5]. Ionic liquids are mainly good in electrical conductivity, solvent transport, and a relatively wide electrochemical window [6]. Configuration of ILs consists of an amphiphilic group with a long chain, hydrophobic tail, and a hydrophilic polar head. ILs contained nitrogen, phosphorus and sulphur which located at the central of cations atom such as imidazolium, pyrrolidinium, quaternary ammonium, pyridinium, piperidinium, sulfonium and quaternary phosphonium. The efficiency of inhibitors dependence on the interaction of functional group and surface of the metal itself. It has been reported that when the concentration of ionic liquid increased, the weight loss of the mild steel decreased due to the formation of film on the surface of the metal [6].

Thus, in this study 1-butyl-3-methylimidazolium chloride [EMIM]Cl ionic liquid was used as the inhibitor for the corrosion protection of mild steel in acidic media. It is believed that imidazolium compounds in this ionic liquid are able to resist corrosion on the mild steel surface [7]. Thus, the effectiveness of $[\mathrm{EMIM}] \mathrm{Cl}$ ionic liquid as inhibitor in different concentrations of sulphuric acid were investigated by using the weight loss measurement method. The effect of [EMIM]Cl ionic liquid concentration on the corrosion process was investigated by determining the weight loss of the mild steel. The ability of [EMIM]Cl to protect 
mild steel in two different types of acid were also elucidated. Fig 1 shows the structure of [EMIM]Cl ionic liquid.

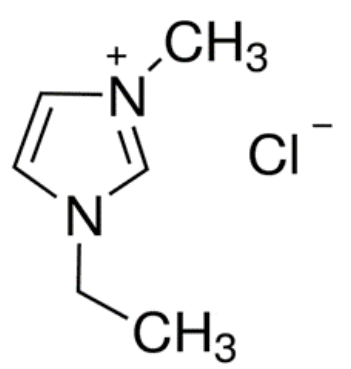

Fig 1: Structure of 1-ethyl-3-methylimidazolium chloride, [EMIM]Cl.

\section{Experimental}

\subsection{Chemicals:}

Ionic liquid, 1-ethyl-3-methylimidazolium chloride [EMIM]Cl was purchased from Sigma Aldrich. Sulphuric acid and hydrochloric used were also purchased from Sigma Aldrich. Acetone and ethanol used as solvent to clean the mild steel surface were purchased form Systerm.

\subsection{Preparation of [EMIM]Cl solution.}

$0.5 \mathrm{M}$ and $0.05 \mathrm{M}$ of [EMIM]Cl were prepared. For $0.05 \mathrm{M}$ of [EMIM]Cl, $1.833 \mathrm{~g}$ of [EMM]Cl was weighed and dissolved with deionized water in $250 \mathrm{~mL}$ volumetric fask. Whilst, $18.328 \mathrm{~g}$ of [EMIM]Cl was weighed and transferred into $250 \mathrm{~mL}$ of volumetric flask for preparation of $0.5 \mathrm{M}$ of [EMIM]Cl. Deionized water was added to dissolve the [EMIM]Cl.

\subsection{Preparation of acid solution.}

Five volumetric flasks $(100 \mathrm{~mL})$ were labelled as $0.05 \mathrm{M}, 0.10 \mathrm{M}$, $0.15 \mathrm{M}, 0.20 \mathrm{M}$ and $0.25 \mathrm{M}$. Then, $0.05 \mathrm{M}$ of sulphuric acid was prepared by measuring $0.275 \mathrm{~mL}$ of sulphuric acid from the stock solution $(18.197 \mathrm{M})$ and transferred it into $100 \mathrm{~mL}$ volumetric flask. Then, deionized water was added into the volumetric flask until it reached the calibrated mark. Then, the same procedure was repeated to prepare other concentrations of sulphuric acid by adding appropriate amount of acid into the volumetric flask for the dilution step. $0.5 \mathrm{M}$ hydrochloric acid were prepared by adding $4.2 \mathrm{~mL}$ of $36 \%$ hydrochloric acids into $100 \mathrm{~mL}$ of volumetric flask and distilled water was added up until the calibrated mark.

\subsection{Preparation of mild steel}

Mild steel coupon $(2 \mathrm{~cm} \mathrm{x} 2 \mathrm{~cm} \times 0.01 \mathrm{~cm})$ was immersed in a 50 $\mathrm{mL}$ beaker containing $10 \mathrm{~mL} 0.05 \mathrm{M}$ sulphuric acid and $10 \mathrm{~mL}$ of $0.05 \mathrm{M}[\mathrm{EMIM}] \mathrm{Cl}$ to observe the corrosion occurrence on the mild steel Surface. Blank sample was polished before being immersed into $50 \mathrm{~mL}$ beaker contained only $10 \mathrm{~mL} 0.05 \mathrm{~mL}$ sulphuric acid. The same procedure was repeated to measure the corrosion effectiveness of mid steel in each concentrations of acid which are $0.10 \mathrm{M}, 0.15 \mathrm{M}, 0.20 \mathrm{M}$ and $0.25 \mathrm{M}$ ) sulphuric acid. The mild steel coupons were immersed at predetermined time (30 minutes, 1 hour, 6 hours, 12 hours and 24 hours). Then, the weight loss value was calculated by using Equation 1:

weight loss, $\Delta \mathrm{W}=\mathbf{m}_{\mathbf{1}}-\mathbf{m}_{\mathbf{2}}$

Where, $\mathbf{m}_{\mathbf{1}}$ and $\mathbf{m}_{\mathbf{2}}$ is the initial and final mass of the specimen putting into the solution [8].

\section{Results and Discussions}

\subsection{Inhibitors performance in different concentrations of sulphuric acid}

The measurement of weight loss was done by weighing of mild steel before and after immersed into different concentrations of acid in the presence of two different concentrations of ionic liquid, which is $0.5 \mathrm{M}$ and $0.05 \mathrm{M}$ of [EMIM]Cl. The final weight of mild steel was measured after 30 minutes, 1 hour, 6 hours, 12 hours and 24 hours respectively. This measurement was done by three replicates of reading to get the average of the weight loss value.

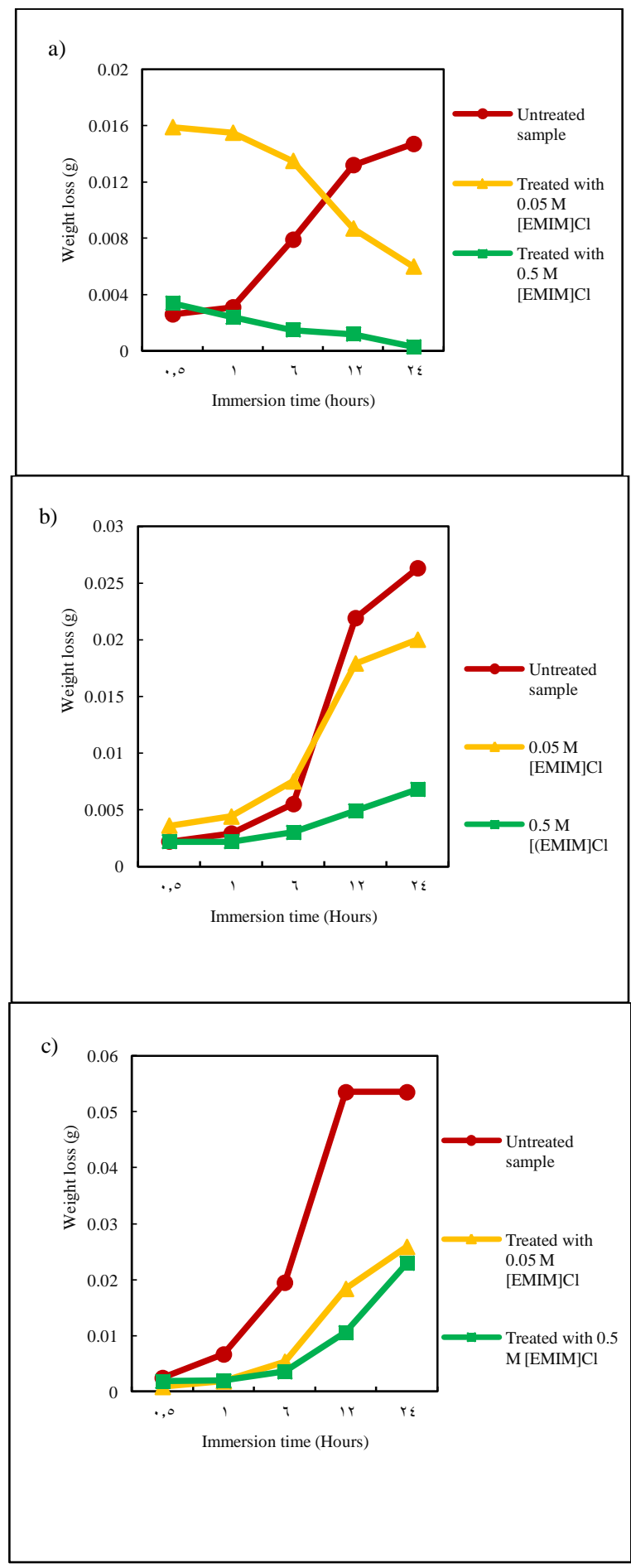




\section{d)}

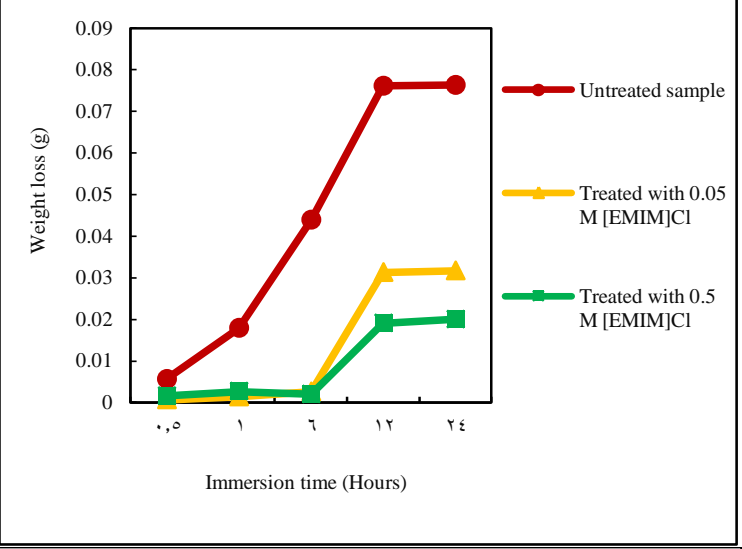

e)

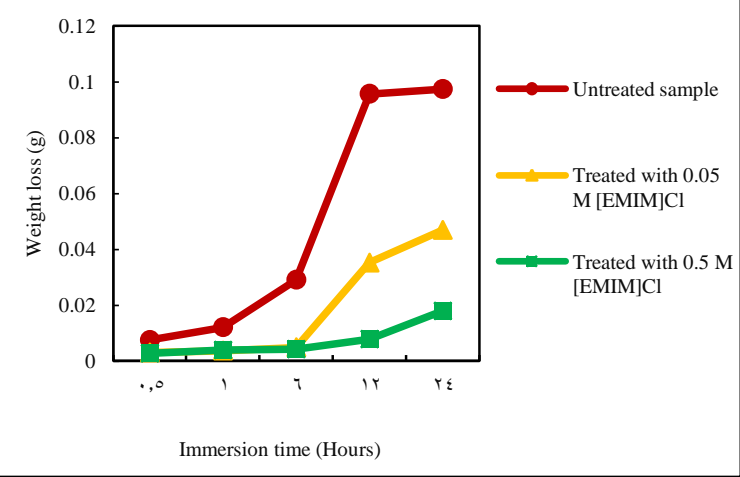

Fig 2: Weight loss value for untreated sample, sample in $0.05 \mathrm{M}$ [EMIM]Cl and $0.5 \mathrm{M}$ [EMIM]Cl in different concentrations of sulphuric acid a) $0.05 \mathrm{M}$, b) $0.1 \mathrm{M}$, c) $0.15 \mathrm{M}$, d) $0.2 \mathrm{M}$ and e) $0.25 \mathrm{M}$.

Fig 2 shows weight loss measurement of untreated sample, sample treated in $0.05 \mathrm{M}$ [EMIM]Cl and sample treated in $0.5 \mathrm{M}$ [EMIM]Cl when exposed in a) $0.05 \mathrm{M}$, b) $0.10 \mathrm{M}$, c) $0.15 \mathrm{M}$, d) $0.20 \mathrm{M}$ and e) $0.25 \mathrm{M}$ sulphuric acid. In this study, $0.05 \mathrm{M}$ sulphuric acid is the lowest concentration acid used as a medium in the weight loss measurement study and $0.25 \mathrm{M}$ is the highest concentration used in this study.

As can be seen in Fig 2 (a), for the untreated sample, the weight loss of the mild steel slowly increased at 30 minutes earlier until 1 hour. After 1 hour, the weight loss of the mild steel increased drastically from $0.0024 \mathrm{~g}$ to $0.0147 \mathrm{~g}$. After addition of $0.05 \mathrm{M}$ [EMIM]Cl ionic liquid the weight loss measurement for the mild steel was slowly decreased from 30 minutes to 24 hours. This shows the effectiveness of 0.05 [EMIM]Cl as a potential inhibitor for corrosion protection. It was believed that the effectiveness of [EMIM] $\mathrm{Cl}$ as inhibitor is due to the presence of $-\mathrm{NH}_{2}$ group which attached to the mild steel surface [9]. In $0.5 \mathrm{M}$ [EMIM]Cl, the corrosion performance of mild steel was better compared to the mild steel treated with $0.05 \mathrm{M}$ of [EMIM]Cl. Fig 2 shows that mild steel treated with $0.5 \mathrm{M}$ [EMIM]Cl revealed the lowest weight loss values even when exposed in the highest $(0.25 \mathrm{M})$ acid concentration used in this experiment (Fig 2 (d)). Weight loss value is directly proportional to corrosion rate value. Yet, lower weight loss value of mild steel treated with $0.5 \mathrm{M}$ [EMIM]Cl ionic liquid revealed that it is a good inhibitor as it able to slow down the corrosion process. Velrani et al. [1], also reported that the corrosion rate decreased considerably with an increase in concentration of the inhibitor. Thus, the higher the concentration of $[\mathrm{EMIM}] \mathrm{Cl}$, the more effective the [EMIM]Cl as inhibitor.

It also can be observed in Fig 2 that the corrosion process occurred slowly with the presence of the ionic liquid. It is believed that the ionic liquid began to form a thin film which act as a protective layer on the mild steel surface [6]. Thus, even the immersion time of the mild steel keep increasing from 30 minutes to 24 hours, the weight loss of the mild steel after treated with ionic liquid was slowly decreased compared to untreated mild steel.

\subsection{Inhibitor performance of $0.5 \mathrm{M}$ and $0.05 \mathrm{M}$ of [EMIM]Cl at 24 hours of exposure.}

Fig 3 shows the weight loss measurement for untreated and treated sample in different concentrations of acid at 24 hours of immersion. Overall, the weight loss rises as the concentration of sulphuric acid increased. Yet, the weight loss value of untreated sample is the highest, showing that the corrosion process occurs rapidly on the mild steel surface. At $0.25 \mathrm{M}$ of sulphuric acid the weight loss of the untreated sample is $0.0974 \mathrm{~g}$. However, when treated with $0.05 \mathrm{M}$ [EMIM]Cl the weight loss of mild steel is lower compared to the weight loss of the untreated sample. The weight loss of mild steel treated with $0.05 \mathrm{M}$ [EMIM]Cl at the highest acid concentration $(0.25 \mathrm{M})$ value is $0.0469 \mathrm{~g}$. Mild steel treated with $0.5 \mathrm{M}$ of [EMIM]Cl revealed the lowest weight loss value which is $0.0179 \mathrm{~g}$. Lower weight loss value of mild steel treated with $0.5 \mathrm{M}$ of [EMIM]Cl suggest that the corrosion process on the mild steel surface occur slowly. Thus, the higher the concentration of [EMIM]Cl used to treat the mild steel, the more effective the [EMIM] Cl can protect the mild steel from being corrode [10].

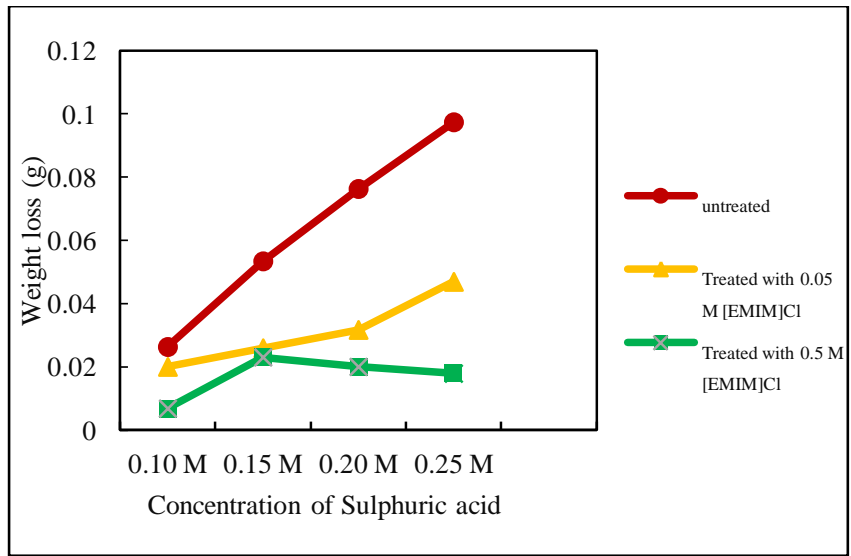

Fig 3: The weight loss (g) of mild steel against different concentrations of sulphuric.

\subsection{Performance of treated mild steel in two different acids solution at 24 hours of exposure.}

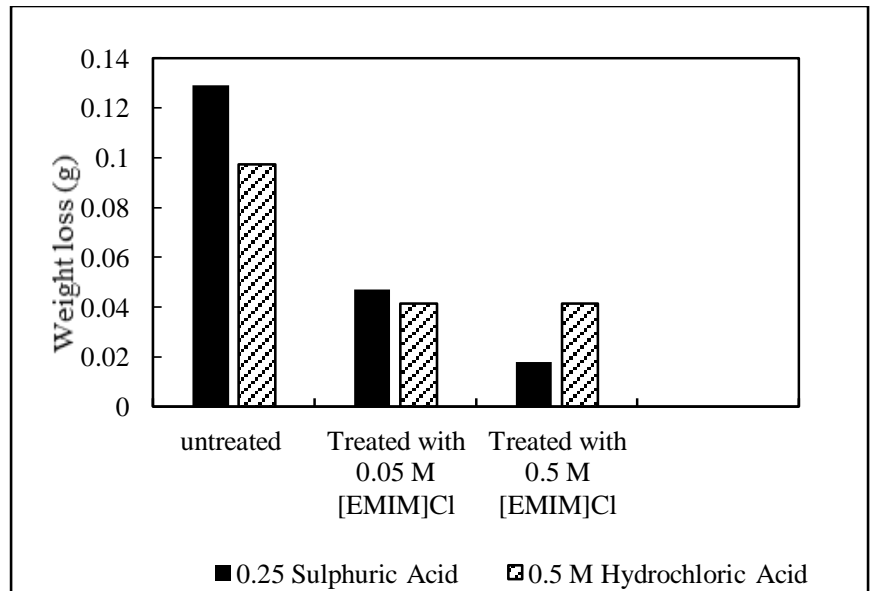

Fig. 4: The weight loss (g) of mild steel against in different acid solution.

The performance of treated mild steel in two different acids solution after 24 hours of exposure was observed. Fig 4 shows the 
weight loss value of the mild steel for untreated mild steel, mild steel treated with $0.05 \mathrm{M}$ [EMIM]Cl and mild steel treated with $0.5 \mathrm{M}$ [EMIM] $\mathrm{Cl}$ when exposed in $0.25 \mathrm{M}$ sulphuric acid and 0.5 $\mathrm{M}$ hydrochloric acid solution. Results revealed that $0.05 \mathrm{M}$ [EMIM]Cl is a better inhibitor when exposed in sulphuric acid solution at 24 hours of exposure. However, in $0.5 \mathrm{M}$ hydrochloric solution, $0.5 \mathrm{M}$ [EMIM]Cl will give better corrosion protection.

\subsection{Scanning electron microscope analysis (SEM)}

Scanning electron microscope (SEM) analysis was conducted to observe the surface morphology of bare mild steel, untreated and treated mild steel. Fig 5 shows SEM image of bare mild steel, untreated mild steel in $0.25 \mathrm{M}$ sulphuric acid at 24 hours and treated mild steel in $0.25 \mathrm{M}$ sulphuric acid at 24 hours at $10000 \mathrm{X}$.

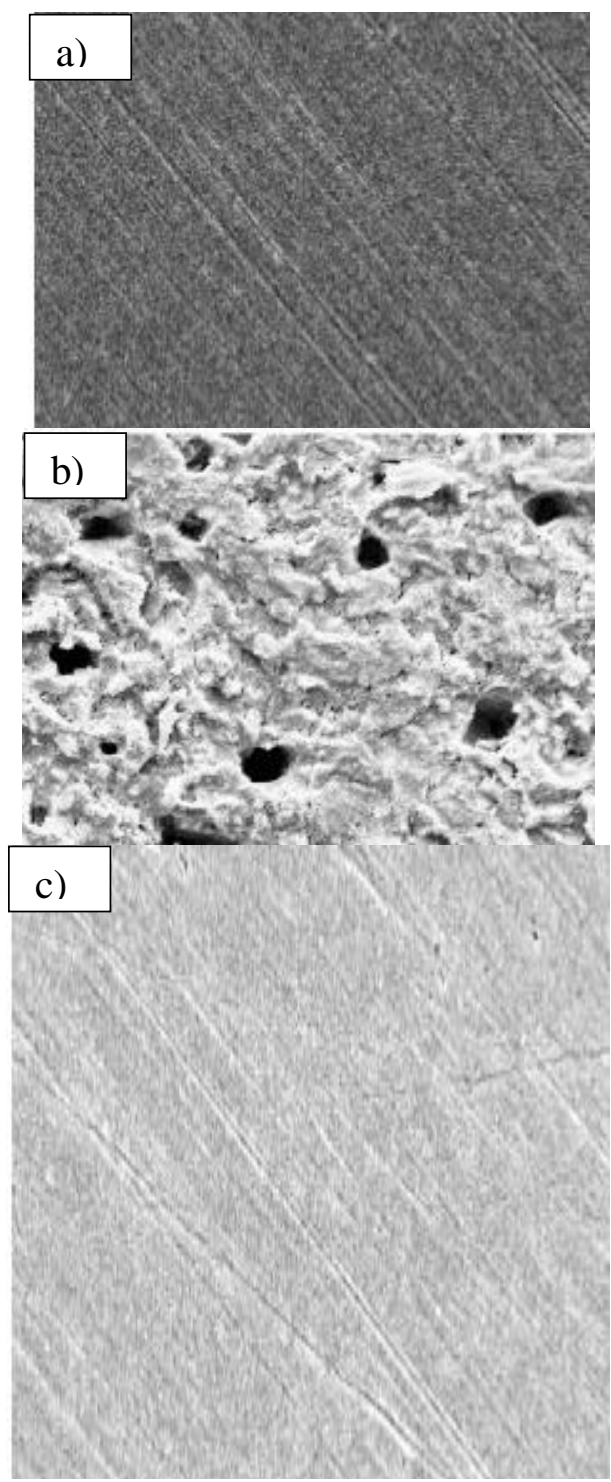

Fig 5: SEM images for a) bare mild steel, b) untreated mild steel in $0.25 \mathrm{M}$ sulphuric acid at 24 hours and c) treated mild steel in $0.25 \mathrm{M}$ sulphuric acid at 24 hours at $10000 \mathrm{X}$

As can be seen in Fig 5 (a), the bare mild steel surface is very smooth surface. However, when the bare mild steel was immersed in $0.25 \mathrm{M}$ sulphuric acid for 24 hours, a rough surface was observed. The rough surface indicates that the mild steel sample was highly corroded. Fig 5 (c) shows the surface morphology of mild steel treated with $0.05 \mathrm{M}$ of [EMIM]Cl. A very smooth and cleaner surface of mild steel can be observed. The 'cleaner' surface indicates the presence of a thin layer of inhibitor film on the mild steel surface.

\section{Conclusion}

In this study, the effectiveness of [EMIM]Cl as corrosion inhibitor in sulfuric acid was investigated. Results revealed that [EMIM]Cl is a good corrosion inhibitor as it can protect the mild steel surface even after 24 hours of immersion. Study showed that, $0.5 \mathrm{M}$ [EMIM]Cl ionic liquid give better protection compared to $0.05 \mathrm{M}$ of $[\mathrm{EMIM}] \mathrm{Cl}$. Thus, higher concentration of [EMIM]Cl give better protection on the mild steel surface compared to lower concentration of [EMIM]Cl. [EMIM]Cl is capable to control the corrosion process even at higher concentration of acid. Study in different acidic media was also conducted and results showed that the type of acid affected the effectiveness of the inhibitor.

\section{Acknowledgement}

The authors would like to express their gratitude to Faculty of Applied Science, UiTM for their support in providing facilities to conduct this research.

\section{References}

[1] Velrani, S., Jeyaprabha, B., Balachandran, V., \& and Prakash, N. (2014). Electrochemical Studies of Mild Steel Corrosion Inhibition by 1-Butyl-3-Methylimidazolium Chloride in 2M $\mathrm{H} 2 \mathrm{SO} 4$ medium. Journal of Applicable Chemistry, 3 (4): 1636-1648.

[2] Bouklah, M., Hammouti, B., Lagrenee, M., \& Bentiss, F., (2006), Thermodynamic properties of 2, 5-bis (4-methoxyphenyl)-1, 3, 4oxadiazole as corrosion inhibitor for mild steel in normal sulphuric acid medium. Corrosion Science, 48, 2831-2842.

[3] Fouda, A., Ismail, M., El-Ewady, G., \& Abousalem, A. (2017). Evaluation of 4-amidinophenyl-2, 2'-bithiophene and its azaanalogue as novel corrosion inhibitors for CS in acidic media: Experimental and theoretical study. Journal of Molecular Liquids, 240, 372-388

[4] Dariva, G. C., \& Galio, F.A. (2014). Corrosion inhibitors - principles, mechanisms and applications. Developments in Corrosion Protection, 365-374.

[5] Seddon, K. R. (1997). Ionic Liquids for Clean Technology. Journal of Chemical Technology \& Biotechnology, 68(4), 351-356.

[6] Scendo, M., \& Uznanska, J. (2011). The Effect of Ionic Liquids on the Corrosion Inhibition of Copper in Acidic Chloride Solutions. International Journal of Corrosion, 2011, 1-13.

[7] Zhang, Q., \& Hua, Y. (2009). Corrosion inhibition of mild steel by alkylimidazolium ionic liquids in hydrochloric acid. Electrochimica Acta, 54(6), 1881-1887.

[8] Ammal, P., R., Prajila, M., Joseph, A, (2017). Effective inhibition of mild steel corrosion in hydrochloric acid using EBIMOT, a 1, 3, 4-oxadiazole derivative bearing a 2-ethylbenzimidazole moiety: Electro analytical, computational and kinetic studies. Egyption Journal of Petroleum,6,1072-1085.

[9] Parsa, M., Hosseini, S. M., Hassani, Z., \& Jamalizadeh, E. (2014). Corrosion protective properties of coatings doped with inhibitors. Anti-Corrosion Methods and Materials, 61(6), 416-422.

[10] Sorkhabi, H.A., \& Es'haghi, M., (2009). Corrosion inhibition of mild steel in acidic media by [BMIm] Br Ionic liquid. Materials Chemistry and Physics, 114, 267-271. 